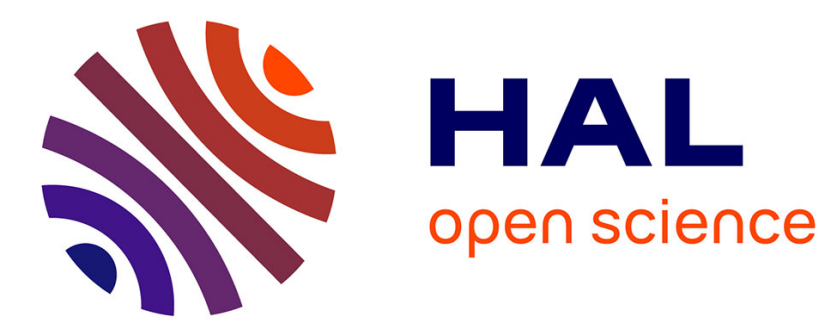

\title{
Two Effective Properties of $\omega$-Rational Functions
}

Olivier Finkel

\section{To cite this version:}

Olivier Finkel. Two Effective Properties of $\omega$-Rational Functions. International Journal of Foundations of Computer Science, 2021, 32 (7), pp.901-920. 10.1142/S0129054121500283 . hal-01870467v4

\section{HAL Id: hal-01870467 \\ https://hal.science/hal-01870467v4}

Submitted on 6 Oct 2021

HAL is a multi-disciplinary open access archive for the deposit and dissemination of scientific research documents, whether they are published or not. The documents may come from teaching and research institutions in France or abroad, or from public or private research centers.
L'archive ouverte pluridisciplinaire HAL, est destinée au dépôt et à la diffusion de documents scientifiques de niveau recherche, publiés ou non, émanant des établissements d'enseignement et de recherche français ou étrangers, des laboratoires publics ou privés. 


\title{
Two Effective Properties of $\omega$-Rational Functions
}

\author{
Olivier Finkel ${ }^{[0000-0002-6461-2941]}$ \\ Institut de Mathématiques de Jussieu - Paris Rive Gauche \\ CNRS et Université de Paris, France. \\ Olivier.Finkel@math.univ-paris-diderot.fr
}

\begin{abstract}
We prove two new effective properties of rational functions over infinite words which are realized by finite state Büchi transducers. Firstly, for each such function $F: \Sigma^{\omega} \rightarrow \Gamma^{\omega}$, one can construct a deterministic Büchi automaton $\mathcal{A}$ accepting a dense $\Pi_{2}^{0}$-subset of $\Sigma^{\omega}$ such that the restriction of $F$ to $L(\mathcal{A})$ is continuous. Secondly, we give a new proof of the decidability of the first Baire class for synchronous $\omega$ rational functions from which we get an extension of this result involving the notion of Wadge classes of regular $\omega$-languages.
\end{abstract}

Keywords: Decision problems - Regular languages of infinite words . Infinitary rational relations $\cdot \omega$-rational functions $\cdot$ Topology $\cdot$ Automatic Baire property - Points of continuity - first Baire class · decidability . Wadge-Wagner class

\section{Introduction}

Infinitary rational relations were first studied by Gire and Nivat $[12,14]$. The $\omega$-rational functions over infinite words, whose graphs are (functional) infinitary rational relations accepted by 2 -tape Büchi automata, have been studied by several authors $[6,1,26,19]$.

In this paper we are mainly interested in topological properties of such $\omega$ rational functions, and in particular in the question of the continuity. Recall that Prieur proved that one can decide whether a given $\omega$-rational function is continuous $[19,20]$. On the other hand, Carton, Finkel and Simonnet proved that one cannot decide whether a given $\omega$-rational function $f$ has at least one point of continuity [5]. Notice that this decision problem is actually $\Sigma_{1}^{1}$-complete, hence highly undecidable [9]. It was also proved in [5] that one cannot decide whether the continuity set of a given $\omega$-rational function $f$ (its set of continuity points) is a regular (respectively, context-free) $\omega$-language. Notice that the situation was shown to be quite different in the case of synchronous functions. It was proved in [5] that if $f: A^{\omega} \rightarrow B^{\omega}$ is an $\omega$-rational synchronous function, then the continuity set $C(f)$ of $f$ is $\omega$-regular. Moreover, if $X \subseteq A^{\omega}$ is a $\Pi_{2}^{0} \omega$-regular language, then $X$ is the continuity set $C(f)$ of some $\omega$-rational synchronous function $f$ of domain $A^{\omega}$. Notice that these previous works on the continuity of $\omega$-rational functions had shown that decision problems in this area may be decidable or not, while it is well known that most problems about regular languages accepted by finite automata are decidable. 
We establish in this paper some new effective properties of rational functions over infinite words. We first prove that regular $\omega$-languages accepted by Büchi or Muller automata satisfy an effective automata-theoretic version of the Baire property which we call here the automatic Baire property. Then we use this result to obtain a new effective property of rational functions over infinite words which are realized by finite state Büchi transducers: for each such function $F$ : $\Sigma^{\omega} \rightarrow \Gamma^{\omega}$, one can construct a deterministic Büchi automaton $\mathcal{A}$ accepting a dense $\Pi_{2}^{0}$-subset of $\Sigma^{\omega}$ such that the restriction of $F$ to this dense set $L(\mathcal{A})$ is continuous.

The reviewer of this paper informed us that in [25, Satz 4] it was actually proved that the $\boldsymbol{\Sigma}_{2}^{0}$ regular $\omega$-languages satisfy the automatic Baire property, although the automatic Baire property was not explicitely stated and used there. It is then easy to infer that regular $\omega$-languages satisfy the automatic Baire property by considering boolean operations.

On the other hand, every $\omega$-rational function $F: \Sigma^{\omega} \rightarrow \Gamma^{\omega}$ is a Borel function, i.e. the inverse image by $F$ of every Borel subset of $\Gamma^{\omega}$ is a Borel subset of $\Sigma^{\omega}$. Recall that the Borel functions $F: \Sigma^{\omega} \rightarrow \Gamma^{\omega}$ from a Cantor space to another Cantor space can be stratified by the Baire hierarchy of the so called Baire class $\xi$ functions, for countable ordinals $\xi[15]$. However every $\omega$-rational function $F: \Sigma^{\omega} \rightarrow \Gamma^{\omega}$ is either of Baire class 0 (continuous), or of Baire class 1 (the inverse image of every open subset of $\Gamma^{\omega}$ is a Borel $\boldsymbol{\Sigma}_{2}^{0}$-subset of $\left.\Sigma^{\omega}\right)$ or of Baire class 2 (the inverse image of every open subset of $\Gamma^{\omega}$ is a Borel $\boldsymbol{\Sigma}_{3}^{0}$-subset of $\left.\Sigma^{\omega}\right)$ see $[3,4]$. It is then very natural to ask whether one can decide whether a given $\omega$-rational function is of Baire class 1. Cagnard and Simonnet proved in [3] that one can decide whether a given synchronous $\omega$-rational function is of Baire class 1 . Their proof used the notion of undergraph and overgraph of a function $F: \Sigma^{\omega} \rightarrow \Gamma^{\omega}$, when the set $\Gamma^{\omega}$ is equipped with the lexicographic ordering. We give here a more direct automata theoretic proof of this result. This way one also obtains an extension of this result. We show that one can decide, for a given synchronous $\omega$-rational function $F: \Sigma^{\omega} \rightarrow \Gamma^{\omega}$, whether the inverse image by $F$ of any basic open subset of $\Gamma^{\omega}$ is in a given Wadge class of the Wadge-Wagner hierarchy of regular $\omega$-languages (where the Wadge-Wagner hierarchy of regular $\omega$-languages is a great refinement of the Borel hierarchy of regular $\omega$-languages).

The first effective property of $\omega$-rational functions proved in this paper firstly appeared in a conference paper published in the proceedings of the 14th International Conference on Language and Automata Theory and Applications, LATA 2020 [10]. The present paper also contains the proof of the second effective property including more results related to the decidability of the first Baire class for $\omega$-rational functions and some refinements of this question. We also give an overview of previous studies and results about continuity of $\omega$-rational functions, and notions of descriptive set theory useful in this paper. We refer the reader to some papers $[25,27,7,29,17]$ for other previous work on Baire property of regular $\omega$-languages. In particular, Staiger established some links between measure theory and Baire category theory in the papers [25, 27], whereas the papers [7, 29] 
connect Baire category theory with the problems of specification and verification encountered in computer science.

The paper is organized as follows. We recall basic notions on automata and on the Borel hierarchy in Section 2. The automatic Baire property for regular $\omega$-languages is proved in Section 3. We prove our first new result on $\omega$-rational functions in Section 4. The decidability of the first Baire class for $\omega$-rational functions and further extensions are studied in Section 5. Some concluding remarks are given in Section 6 .

\section{Recall of basic notions}

We assume the reader to be familiar with the theory of formal $(\omega)$-languages $[28,26,18]$. We recall some usual notations of formal language theory.

When $\Sigma$ is a finite alphabet, a non-empty finite word over $\Sigma$ is any sequence $x=a_{1} \ldots a_{k}$, where $a_{i} \in \Sigma$ for $i=1, \ldots, k$, and $k$ is an integer $\geq 1$. The length of $x$ is $|x|=k$. The set of finite words (including the empty word $\varepsilon$ whose length is zero) over $\Sigma$ is denoted $\Sigma^{\star}$.

The first infinite ordinal is $\omega$. An $\omega$-word over $\Sigma$ is an $\omega$-sequence $a_{1} \ldots a_{n} \ldots$, where for all integers $i \geq 1, a_{i} \in \Sigma$. When $\sigma$ is an $\omega$-word over $\Sigma$, we write $\sigma=\sigma(1) \sigma(2) \ldots \sigma(n) \ldots$, where for all $i, \sigma(i) \in \Sigma$, and $\sigma[n]=\sigma(1) \sigma(2) \ldots \sigma(n)$.

The usual concatenation product of two finite words $u$ and $v$ is denoted $u \cdot v$ and sometimes just $u v$. This product is extended to the product of a finite word $u$ and an $\omega$-word $v$. The infinite word $u \cdot v$ is then the $\omega$-word such that: $(u \cdot v)(k)=u(k)$ if $k \leq|u|$, and $(u \cdot v)(k)=v(k-|u|)$ if $k>|u|$. The concatenation product can be extended in an obvious way to the concatenation of an infinite sequence of finite words. The concatenation of a set $U$ of finite words with a set $V$ of infinite words is the set of infinite words $U \cdot V=\{u . v \mid u \in U$ and $v \in V\}$. If $u$ is a finite word and $V$ is a set of infinite words then $u \cdot V=\{u \cdot v \mid v \in V\}$.

The prefix relation is denoted $\sqsubseteq$ : a finite word $u$ is a prefix of a finite word $v$ (respectively, an infinite word $v$ ), denoted $u \sqsubseteq v$, if and only if there exists a finite word $w$ (respectively, an infinite word $w$ ), such that $v=u \cdot w$. For a finite or infinite word $v$ we denote $\operatorname{Pref}(v)$ the set of prefixes of $v$, and for a set $L$ of finite or infinite words we denote $\operatorname{Pref}(L)=\bigcup_{v \in L} \operatorname{Pref}(v)$.

The set of $\omega$-words over the alphabet $\Sigma$ is denoted by $\Sigma^{\omega}$. An $\omega$-language over an alphabet $\Sigma$ is a subset of $\Sigma^{\omega}$.

Definition 1. : A finite state machine (FSM) is a quadruple $\mathcal{M}=\left(K, \Sigma, \delta, q_{0}\right)$, where $K$ is a finite set of states, $\Sigma$ is a finite input alphabet, $q_{0} \in K$ is the initial state and $\delta$ is a mapping from $K \times \Sigma$ into $2^{K}$. A FSM is called deterministic iff: $\delta: K \times \Sigma \rightarrow\{\{q\} \mid q \in K\}$. (As usual, by a clear identification, we might consider in that case that $\delta: K \times \Sigma \rightarrow K)$.

$A$ Büchi automaton (BA) is a 5-tuple $\mathcal{A}=\left(K, \Sigma, \delta, q_{0}, F\right)$ where $\mathcal{M}=$ $\left(K, \Sigma, \delta, q_{0}\right)$ is a finite state machine and $F \subseteq K$ is the set of final states.

$A$ Muller automaton (MA) is a 5-tuple $\mathcal{A}=\left(K, \Sigma, \delta, q_{0}, \mathcal{F}\right)$ where $\mathcal{M}=$ $\left(K, \Sigma, \delta, q_{0}\right)$ is a FSM and $\mathcal{F} \subseteq 2^{K}$ is the collection of designated state sets. 
A Büchi or Muller automaton is said to be deterministic if the associated FSM is deterministic.

We assume the reader to be familiar with the basic theory of Büchi and Muller automata. For a Büchi or Muller automaton $\mathcal{A}$ we denote $L(\mathcal{A})$ the $\omega$-language accepted by $\mathcal{A}$. An $\omega$-language is regular iff it is accepted by a Büchi or Muller automaton. Recall that every regular $\omega$-language is accepted by a deterministic Muller automaton.

A way to study the complexity of $\omega$-languages accepted by various automata is to study their topological complexity.

We assume the reader to be familiar with basic notions of topology which may be found in $[15,26,18]$. If $X$ is a finite alphabet containing at least two letters, then the set $X^{\omega}$ of infinite words over $X$ may be equipped with the product topology of the discrete topology on $X$. This topology is induced by a natural metric which is called the prefix metric and is defined as follows. For $u, v \in X^{\omega}$ and $u \neq v$ let $\delta(u, v)=2^{-l_{\operatorname{pref}(u, v)}}$ where $l_{\operatorname{pref}(u, v)}$ is the first integer $n$ such that the $u(n+1)$ is different from $v(n+1)$. The topological space $X^{\omega}$ is a Cantor space. The open sets of $X^{\omega}$ are the sets of the form $W \cdot X^{\omega}$, where $W \subseteq X^{\star}$. A set $L \subseteq X^{\omega}$ is a closed set iff its complement $X^{\omega}-L$ is an open set. Closed sets are characterized by the following:

Proposition 2. A set $L \subseteq X^{\omega}$ is a closed set of $X^{\omega}$ iff for every $\sigma \in X^{\omega}$, $\operatorname{Pref}(\sigma) \subseteq \operatorname{Pref}(L)$ implies that $\sigma \in L$.

Define now the next classes of the Borel hierarchy:

Definition 3. The classes $\boldsymbol{\Sigma}_{n}^{0}$ and $\boldsymbol{\Pi}_{n}^{0}$ of the Borel Hierarchy on the topological space $X^{\omega}$ are defined as follows: $\boldsymbol{\Sigma}_{1}^{0}$ is the class of open sets of $X^{\omega}, \Pi_{1}^{0}$ is the class of closed sets of $X^{\omega}$. And for any integer $n \geq 1: \boldsymbol{\Sigma}_{n+1}^{0}$ is the class of countable unions of $\boldsymbol{\Pi}_{n}^{0}$-subsets of $X^{\omega}$, and $\boldsymbol{\Pi}_{n+1}^{0}$ is the class of countable intersections of $\boldsymbol{\Sigma}_{n}^{0}$-subsets of $X^{\omega}$.

It turns out that there is a characterization of $\Pi_{2}^{0}$-subsets of $X^{\omega}$, involving the notion of $W^{\delta}$ which we now recall, see $[26,18]$.

Definition 4. For $W \subseteq X^{\star}$, we set: $W^{\delta}=\left\{\sigma \in X^{\omega} \mid \exists^{\infty} i\right.$ such that $\left.\sigma[i] \in W\right\}$. $\left(\sigma \in W^{\delta}\right.$ iff $\sigma$ has infinitely many prefixes in $W$.)

Then we can state the following proposition.

Proposition 5. A subset $L$ of $X^{\omega}$ is a $\Pi_{2}^{0}$-subset of $X^{\omega}$ iff there exists a set $W \subseteq X^{\star}$ such that $L=W^{\delta}$.

Recall that a regular $\omega$-language is in the class $\Pi_{2}^{0}$ iff it is accepted by a deterministic Büchi automaton.

We also recall that usual operations on regular $\omega$-languages (as Boolean operations, interior, closure or projection) are effective: one can construct the respective automata from given ones. 


\section{The automatic Baire property}

In this section we are going to prove an automatic version of the result stating that every Borel (and even every analytic) set has the Baire property.

We firstly recall some basic definitions about meager sets, see [15]. In a topological space $\mathcal{X}$, a set $A \subseteq \mathcal{X}$ is said to be nowhere dense if its closure $\bar{A}$ has empty interior, i.e. $\operatorname{Int}(\bar{A})=\emptyset$. A set $A \subseteq \mathcal{X}$ is said to be meager if it is the union of countably many nowhere dense sets, or equivalently if it is included in a countable union of closed sets with empty interiors. This means that $A$ is meager if there exist countably many closed sets $A_{n}, n \geq 1$, such that $A \subseteq \bigcup_{n>1} A_{n}$ where for every integer $n \geq 1$, $\operatorname{Int}\left(A_{n}\right)=\emptyset$. A set is comeager if its complement is meager, i.e. if it contains the intersection of countably many dense open sets. Notice that the notion of a meager set is a notion of a small set, while the notion of a comeager set is a notion of a big set.

Recall that a Baire space is a topological space $\mathcal{X}$ in which every intersection of countably many dense open sets is dense, or equivalently in which every countable union of closed sets with empty interiors has also an empty interior. It is well known that every Cantor space $\Sigma^{\omega}$ is a Baire space. In the sequel we will consider only Cantor spaces.

We now recall the notion of Baire property. For any sets $A, B \subseteq \Sigma^{\omega}$, we denote by $A \Delta B$ the symmetric difference of $A$ and $B$, and we write $A={ }^{\star} B$ if and only if $A \Delta B$ is meager.

Definition 6. A set $A \subseteq \Sigma^{\omega}$ has the Baire property (BP) if there exists an open set $U \subseteq \Sigma^{\omega}$ such that $A={ }^{\star} U$.

An important result of descriptive set theory is the following result, see $[15$, page 47].

Theorem 7. Every Borel set of a Cantor space has the Baire property.

We are going to prove an automatic version of the above theorem. We first give the following definition.

Definition 8. Let $L=L(\mathcal{A}) \subseteq \Sigma^{\omega}$, where $\mathcal{A}$ is a Büchi or Muller automaton. The $\omega$-language $L$ is said to have the automatic Baire property if one can construct from $\mathcal{A}$ some Büchi automata $\mathcal{B}$ and $\mathcal{C}$ such that $L(\mathcal{B}) \subseteq \Sigma^{\omega}$ is open, $L(\mathcal{C}) \subseteq \Sigma^{\omega}$ is a countable union of closed sets with empty interior, i.e. a meager $\boldsymbol{\Sigma}_{2}^{0}$-set, and $L(\mathcal{A}) \Delta L(\mathcal{B}) \subseteq L(\mathcal{C})$.

We already know that the regular $\omega$-languages have the Baire property since they are Borel. We now state the following theorem which gives an automatic version of this result.

Theorem 9. Every regular $\omega$-language $L=L(\mathcal{A})$, where $\mathcal{A}$ is a given Büchi or Muller automaton, has the automatic Baire property.

In order to prove this result, we first state the following lemmas. 
Lemma 10. [Staiger, see [25, Satz 4]] Every regular $\omega$-language which is a $\boldsymbol{\Sigma}_{2}^{0}$ set has the automatic Baire property.

Proof. Let $L$ be a regular $\omega$-language which is a $\boldsymbol{\Sigma}_{2}^{0}$-set. The result follows from the fact that $L \Delta \operatorname{Int}(L)=L \backslash \operatorname{Int}(L)$ is meager and regular.

Lemma 11. Let $L \subseteq \Sigma^{\omega}$ be a regular $\omega$-language which has the automatic Baire property. Then its complement $\Sigma^{\omega} \backslash L$ has also the automatic Baire property.

Proof. Assume that the regular $\omega$-language $L=L(\mathcal{A})$ has the automatic Baire property, and that $L \Delta O \subseteq M$ with $\mathrm{O}, \mathrm{M}$ regular, $O$ open and $M$ meager and $\Sigma_{2}^{0}$. Notice first that $\left(\Sigma^{\omega} \backslash L\right) \Delta\left(\Sigma^{\omega} \backslash O\right)=L \Delta O$. Then we have the following inclusion:

$$
\left(\Sigma^{\omega} \backslash L\right) \Delta \operatorname{Int}\left(\Sigma^{\omega} \backslash O\right) \subseteq M \cup\left[\left(\Sigma^{\omega} \backslash O\right) \backslash \operatorname{Int}\left(\Sigma^{\omega} \backslash O\right)\right]
$$

which easily implies that $\left(\Sigma^{\omega} \backslash L\right)$ has the automatic Baire property.

Notice that this also implies, from Lemma 10, that the regular $\omega$-languages in the Borel class $\Pi_{2}^{0}$ have the automatic Baire property.

Lemma 12. The class of regular $\omega$-languages having the automatic Baire property is closed under finite union and under finite intersection.

Proof. We first consider the finite union operation. Assume $L \Delta O \subseteq M$ and $L^{\prime} \Delta O^{\prime} \subseteq M^{\prime}$, where $L, L^{\prime}$ regular, $O, O^{\prime}$ open and regular, $M, M^{\prime}$ regular, meager, and $\boldsymbol{\Sigma}_{2}^{0}$. The result follows easily from the inclusion $\left(L \cup L^{\prime}\right) \Delta\left(O \cup O^{\prime}\right) \subseteq$ $(L \Delta O) \cup\left(L^{\prime} \Delta O^{\prime}\right) \subseteq M \cup M^{\prime}$.

The case of the intersection of two regular $\omega$-languages now follows from the case of the union and the case of the complement proved in Lemma 11.

End of Proof of Theorem 9. We now return to the general case of a regular $\omega$-language $L(\mathcal{A}) \subseteq \Sigma^{\omega}$, where $\mathcal{A}=\left(K, \Sigma, \delta, q_{0}, \mathcal{F}\right)$ is a deterministic Muller automaton. Recall that $\mathcal{F} \subseteq 2^{K}$ is here the collection of designated state sets. For each state $q \in K$, we now denote by $\mathcal{A}^{(q)}$ the automaton $\mathcal{A}$ but viewed as a (deterministic) Büchi automaton with the single accepting state $q$, i.e. $\mathcal{A}^{(q)}=$ $\left(K, \Sigma, \delta, q_{0},\{q\}\right)$. We know that the languages $L\left(\mathcal{A}^{(q)}\right)$ are Borel $\boldsymbol{\Pi}_{2}^{0}$-sets and thus satisfy the automatic Baire property by Lemmas 10 and 11 . Moreover we have the following equality:

$$
L(\mathcal{A})=\bigcup_{F \in \mathcal{F}}\left[\bigcap_{q \in F} L\left(\mathcal{A}^{(q)}\right) \backslash \bigcup_{q \notin F} L\left(\mathcal{A}^{(q)}\right)\right]
$$

This implies, from the previous lemmas about the preservation of the automatic Baire property by Boolean operations, that the $\omega$-language $L$ has the automatic Baire property. 


\section{An application to $\omega$-rational functions}

\subsection{Infinitary rational relations}

We now recall the definition of infinitary rational relations, via definition by Büchi transducers:

Definition 13. A 2-tape Büchi automaton is a 6-tuple $\mathcal{T}=\left(K, \Sigma, \Gamma, \Delta, q_{0}, F\right)$, where $K$ is a finite set of states, $\Sigma$ and $\Gamma$ are finite sets called the input and the output alphabets, $\Delta$ is a finite subset of $K \times(\Sigma \cup \varepsilon) \times(\Gamma \cup \varepsilon) \times K$ called the set of transitions, $q_{0}$ is the initial state, and $F \subseteq K$ is the set of accepting states. $A$ computation $\mathcal{C}$ of the automaton $\mathcal{T}$ is an infinite sequence of consecutive transitions

$$
\left(q_{0}, u_{1}, v_{1}, q_{1}\right),\left(q_{1}, u_{2}, v_{2}, q_{2}\right), \ldots\left(q_{i-1}, u_{i}, v_{i}, q_{i}\right),\left(q_{i}, u_{i+1}, v_{i+1}, q_{i+1}\right), \ldots
$$

The computation is said to be successful iff there exists a final state $q_{f} \in F$ and infinitely many integers $i \geq 0$ such that $q_{i}=q_{f}$. The input word and output word of the computation are respectively $u=u_{1} \cdot u_{2} \cdot u_{3} \ldots$ and $v=v_{1} \cdot v_{2} \cdot v_{3} \ldots$ The input and the output words may be finite or infinite. The infinitary rational relation $R(\mathcal{T}) \subseteq \Sigma^{\omega} \times \Gamma^{\omega}$ accepted by the 2 -tape Büchi automaton $\mathcal{T}$ is the set of pairs $(u, v) \in \Sigma^{\omega} \times \Gamma^{\omega}$ such that $u$ and $v$ are the input and the output words of some successful computation $\mathcal{C}$ of $\mathcal{T}$.

The 2-tape Büchi automaton $\mathcal{T}=\left(K, \Sigma, \Gamma, \Delta, q_{0}, F\right)$ is said to be synchronous if the set of transitions $\Delta$ is a finite subset of $K \times \Sigma \times \Gamma \times K$, i.e. if each transition is labelled with a pair $(a, b) \in \Sigma \times \Gamma$. An infinitary rational relation recognized by a synchronous 2-tape Büchi automaton is in fact, via the natural identification of $\Sigma^{\omega} \times \Gamma^{\omega}$ with $(\Sigma \times \Gamma)^{\omega}$, an $\omega$-language over the product alphabet $\Sigma \times \Gamma$ which is accepted by a Büchi automaton. It is called a synchronous infinitary rational relation. An infinitary rational relation is said to be asynchronous if it can not be recognized by any synchronous 2-tape Büchi automaton.

Remark 14. In the above definition, we could have defined the set of transitions $\Delta$ as a subset of $K \times \Sigma^{\star} \times \Gamma^{\star} \times K$. We have chosen to define $\Delta$ as a finite subset of $K \times(\Sigma \cup \varepsilon) \times(\ulcorner\cup \varepsilon) \times K$ to simplify the proofs. However this is done without loss of generality because it is easy to see that this convention does not change the class of infinitary rational relations.

If $R(\mathcal{T}) \subseteq \Sigma^{\omega} \times \Gamma^{\omega}$ is an infinitary rational relation recognized by the 2-tape Büchi automaton $\mathcal{T}$ then we denote

$$
\operatorname{Dom}(R(\mathcal{T}))=\left\{u \in \Sigma^{\omega} \mid \exists v \in \Gamma^{\omega} \quad(u, v) \in R(\mathcal{T})\right\}
$$

and

$$
\operatorname{Im}(R(\mathcal{T}))=\left\{v \in \Gamma^{\omega} \mid \exists u \in \Sigma^{\omega}(u, v) \in R(\mathcal{T})\right\} .
$$

It is well known that, for each infinitary rational relation $R(\mathcal{T}) \subseteq \Sigma^{\omega} \times \Gamma^{\omega}$, the sets $\operatorname{Dom}(R(\mathcal{T}))$ and $\operatorname{Im}(R(\mathcal{T}))$ are regular $\omega$-languages and that one can construct, from the Büchi transducer $\mathcal{T}$, some (non-deterministic) Büchi automata $\mathcal{A}$ and $\mathcal{B}$ accepting the $\omega$-languages $\operatorname{Dom}(R(\mathcal{T}))$ and $\operatorname{Im}(R(\mathcal{T}))$.

Recall now the following undecidability result of Frougny and Sakarovitch. 
Theorem 15 ([11]). One cannot decide whether a given infinitary rational relation is synchronous.

We proved in [8] that many decision problems about infinitary rational relations are highly undecidable. In fact many of them, like the universality problem, the equivalence problem, the inclusion problem, the cofiniteness problem, the unambiguity problem, are $\Pi_{2}^{1}$-complete, hence located at the second level of the analytical hierarchy.

\subsection{Continuity of $\omega$-rational functions}

Recall that an infinitary rational relation $R(\mathcal{T}) \subseteq \Sigma^{\omega} \times \Gamma^{\omega}$ is said to be functional iff it is the graph of a function, i.e. iff

$$
[\forall x \in \operatorname{Dom}(R(\mathcal{T})) \quad \exists ! y \in \operatorname{Im}(R(\mathcal{T})) \quad(x, y) \in R(\mathcal{T})]
$$

Then the functional relation $R(\mathcal{T})$ defines an $\omega$-rational (partial) function $F_{\mathcal{T}}$ : $\operatorname{Dom}(R(\mathcal{T})) \rightarrow \Gamma^{\omega}$ by: for each $u \in \operatorname{Dom}(R(\mathcal{T})), F_{\mathcal{T}}(u)$ is the unique $v \in \Gamma^{\omega}$ such that $(u, v) \in R(\mathcal{T})$.

An $\omega$-rational (partial) function $f: \Sigma^{\omega} \rightarrow \Gamma^{\omega}$ is said to be synchronous if there is a synchronous 2-tape Büchi automaton $\mathcal{T}$ such that $f=F_{\mathcal{T}}$.

An $\omega$-rational (partial) function $f: \Sigma^{\omega} \rightarrow \Gamma^{\omega}$ is said to be asynchronous if there is no synchronous 2-tape Büchi automaton $\mathcal{T}$ such that $f=F_{\mathcal{T}}$.

Recall the following previous decidability result.

Theorem 16 (Gire [13]). One can decide whether an infinitary rational relation recognized by a given 2-tape Büchi automaton $\mathcal{T}$ is a functional infinitary rational relation.

We refer the interested reader to the proof of this theorem in [13] where a characterization of functional infinitary rational relations is given.

It is very natural to consider the notion of continuity for $\omega$-rational functions defined by 2-tape Büchi automata.

We recall that a function $f: \operatorname{Dom}(f) \subseteq \Sigma^{\omega} \rightarrow \Gamma^{\omega}$, whose domain is $\operatorname{Dom}(f)$, is said to be continuous at point $x \in \operatorname{Dom}(f)$ if :

$$
\forall n \geq 1 \quad \exists k \geq 1 \quad \forall y \in \operatorname{Dom}(f) \quad\left[\delta(x, y)<2^{-k} \Rightarrow \delta(f(x), f(y))<2^{-n}\right]
$$

The continuity set $C(f)$ of the function $f$ is the set of points of continuity of $f$. Notice that the continuity set $C(f)$ of a function $f: \Sigma^{\omega} \rightarrow \Gamma^{\omega}$ is always a Borel $\boldsymbol{\Pi}_{2}^{0}$-subset of $\Sigma^{\omega}$, see [5].

The function $f$ is said to be continuous if it is continuous at every point $x \in \operatorname{Dom}(f)$, i. e. if $C(f)=\operatorname{Dom}(f)$.

Prieur proved the following decidability result.

Theorem 17 (Prieur [19, 20]). One can decide whether a given w-rational function is continuous. 
On the other hand the following undecidability result was proved in [5].

Theorem 18 (see [5]). One cannot decide whether a given $\omega$-rational function $f$ has at least one point of continuity.

The exact complexity of this undecidable problem was given in [9]. It is $\Sigma_{1}^{1}$ complete to determine whether a given $\omega$-rational function $f$ has at least one point of continuity.

We now consider the continuity set of an $\omega$-rational function and its possible complexity. The following undecidability result was proved in [5].

Theorem 19 (see [5]). One cannot decide whether the continuity set of a given $\omega$-rational function $f$ is a regular (respectively, context-free) $\omega$-language.

The situation is quite different in the case of synchronous functions. The following results were proved in [5].

Theorem 20 ([5]). Let $f: A^{\omega} \rightarrow B^{\omega}$ be a rational synchronous function. The continuity set $C(f)$ of $f$ is rational.

Theorem 21 ([5]). Let $X$ be a rational $\Pi_{2}^{0}$ subset of $A^{\omega}$. Then $X$ is the continuity set $C(f)$ of some rational synchronous function $f$ of domain $A^{\omega}$.

We are now going to prove another effective result about $\omega$-rational functions.

We first recall the following result of descriptive set theory, in the particular case of Cantor spaces $\Sigma^{\omega}$ and $\Gamma^{\omega}$. A Borel function $f: \Sigma^{\omega} \rightarrow \Gamma^{\omega}$ is a function for which the inverse image of any Borel subset of $\Gamma^{\omega}$, or equivalently of any open set of $\Gamma^{\omega}$, is a Borel subset of $\Sigma^{\omega}$.

Theorem 22 (see Theorem 8.38 of [15]). Let $\Sigma$ and $\Gamma$ be two finite alphabets and $f: \Sigma^{\omega} \rightarrow \Gamma^{\omega}$ be a Borel function. Then there is a dense $\Pi_{2}^{0}$-subset $G$ of $\Sigma^{\omega}$ such that the restriction of $f$ to $G$ is continuous.

We now state an automatic version of this theorem.

Theorem 23. Let $\Sigma$ and $\Gamma$ be two finite alphabets and $f: \Sigma^{\omega} \rightarrow \Gamma^{\omega}$ be an $\omega$-rational function. Then one can construct, from a 2-tape Büchi automaton accepting the graph of the function $f$, a deterministic Büchi automaton accepting a dense $\Pi_{2}^{0}$-subset $G$ of $\Sigma^{\omega}$ such that the restriction of $f$ to $G$ is continuous.

Proof. Let $\Sigma$ and $\Gamma$ be two finite alphabets and $f: \Sigma^{\omega} \rightarrow \Gamma^{\omega}$ be an $\omega$-rational function whose graph is accepted by a 2 -tape Büchi automaton $\mathcal{A}=$ $\left(K, \Sigma, \Gamma, \Delta, q_{0}, F\right)$.

Notice that one can also consider the 2-tape automaton $\mathcal{A}$ reading pairs of finite words $(v, u) \in \Sigma^{\star} \times \Gamma^{\star}$. A partial computation of the 2-tape automaton $\mathcal{A}$ reading such a pair $(v, u)$ is simply a finite sequence of consecutive transitions

$$
\left(q_{0}, a_{1}, b_{1}, q_{1}\right),\left(q_{1}, a_{2}, b_{2}, q_{2}\right), \ldots\left(q_{i-1}, a_{i}, b_{i}, q_{i}\right),\left(q_{i}, a_{i+1}, b_{i+1}, q_{i+1}\right)
$$


such that $v=a_{1} a_{2} \ldots a_{i+1}$ and $u=b_{1} b_{2} \ldots b_{i+1}$. This computation ends in state $q_{i+1}$.

We assume that we have an effective enumeration of the finite words over the alphabet $\Gamma$ given by $\left(u_{n}\right)_{n \geq 1}, u_{n} \in \Gamma^{\star}$. For $q \in K$ we also denote $\mathcal{A}_{q}$ the automaton $\mathcal{A}$ in which we have changed the initial state so that the initial state of $\mathcal{A}_{q}$ is $q$ instead of $q_{0}$.

Let us now consider the basic open set of the space $\Gamma^{\omega}$ given by $U_{n}=u_{n} \cdot \Gamma^{\omega}$. We first describe $f^{-1}\left(U_{n}\right)$. An $\omega$-word $x \in \Sigma^{\omega}$ belongs to the set $f^{-1}\left(U_{n}\right)$ iff $x$ can be written in the form $x=v \cdot y$ for some words $v \in \Sigma^{\star}$ and $y \in \Sigma^{\omega}$, and there is a partial computation of the automaton $\mathcal{A}$ reading $\left(v, u_{n}\right)$ for which $\mathcal{A}$ is in state $q$ after having read the initial pair $\left(v, u_{n}\right) \in \Sigma^{\star} \times \Gamma^{\star}$ (where the finite words $v$ and $u_{n}$ might have different lengths if the automaton $\mathcal{A}$ is not synchronous), and $y \in \operatorname{Dom}\left(R\left(\mathcal{A}_{q}\right)\right)$. Recall that $R\left(\mathcal{A}_{q}\right) \subseteq \Sigma^{\omega} \times \Gamma^{\omega}$ is an infinitary rational relation and that $\operatorname{Dom}\left(R\left(\mathcal{A}_{q}\right)\right)$, which will be denoted $L_{q}$, is then a regular $\omega$ language accepted by a deterministic Muller automaton. We also denote $T\left(u_{n}, q\right)$ the set of finite words $v$ over $\Sigma$ such that the automaton $\mathcal{A}$ may be in state $q$ after having read the initial pair $\left(v, u_{n}\right) \in \Sigma^{\star} \times \Gamma^{\star}$. Then the following equality holds:

$$
f^{-1}\left(U_{n}\right)=\bigcup_{q \in K} T\left(u_{n}, q\right) \cdot L_{q}
$$

We can now apply the automatic Baire property stated in the above Theorem 9 . Then for each regular $\omega$-language $L_{q}$, one can construct a deterministic Muller automaton accepting an open set $O_{q}$ and a deterministic Muller automaton accepting a countable union $W_{q}$ of closed sets with empty interiors, such that for each $q \in K$,

$$
L_{q} \Delta O_{q} \subseteq W_{q}
$$

Now we set

$$
V_{n}=\bigcup_{q \in K} T\left(u_{n}, q\right) \cdot O_{q} \quad \text { and } \quad F_{n}=\bigcup_{q \in K} T\left(u_{n}, q\right) \cdot W_{q}
$$

Notice that each set $T\left(u_{n}, q\right)$ is countable and that for each finite word $u \in$ $T\left(u_{n}, q\right)$ it is easy to see that the set $u \cdot O_{q}$ is open and that the set $u \cdot W_{q}$ is a countable union of closed sets with empty interiors. Thus it is easy to see that $V_{n}$ is open, and that $F_{n}$ is a countable union of closed sets with empty interiors. Moreover it is easy to see that $V_{n}$ and $F_{n}$ are regular $\omega$-languages since each set $T\left(u_{n}, q\right)$ is a regular language of finite words over the alphabet $\Sigma$. Moreover it holds that:

$$
f^{-1}\left(U_{n}\right) \Delta V_{n} \subseteq F_{n}
$$

This follows from: (1) for each $u \in T\left(u_{n}, q\right)$ it holds that $\left(u \cdot L_{q}\right) \Delta\left(u \cdot O_{q}\right) \subseteq\left(u \cdot W_{q}\right)$ and (2) if for all $i \in I$, where $I$ is a countable set, $L_{i} \Delta O_{i} \subseteq M_{i}$, then we have

$$
\left(\bigcup_{i \in I} L_{i}\right) \Delta\left(\bigcup_{i \in I} O_{i}\right) \subseteq \bigcup_{i \in I} L_{i} \Delta O_{i} \subseteq\left(\bigcup_{i \in I} M_{i}\right)
$$


We now prove that $F=\bigcup_{n \geq 1} F_{n}$ is itself a regular $\omega$-language. It holds that

$$
F=\bigcup_{n \geq 1} F_{n}=\bigcup_{n \geq 1} \bigcup_{q \in K} T\left(u_{n}, q\right) \cdot W_{q}=\bigcup_{q \in K} \bigcup_{n \geq 1} T\left(u_{n}, q\right) \cdot W_{q}
$$

Consider now the 2-tape automaton $\mathcal{B}_{q}$ which is like the 2-tape automaton $\mathcal{A}$ but reads only pairs of finite words in $\Sigma^{\star} \times \Gamma^{\star}$ and has the state $q$ as unique accepting state. Let then $\mathcal{C}_{q}$ be a finite automaton which reads only finite words over the alphabet $\Sigma$ and such that $L\left(\mathcal{C}_{q}\right)=\operatorname{Proj}_{\Sigma^{\star}}\left(L\left(\mathcal{B}_{q}\right)\right)$ is the projection of the language $L\left(\mathcal{B}_{q}\right)$ on $\Sigma^{\star}$. Now it holds that:

$$
F=\bigcup_{n \geq 1} F_{n}=\bigcup_{q \in K} \bigcup_{n \geq 1} T\left(u_{n}, q\right) \cdot W_{q}=\bigcup_{q \in K} L\left(\mathcal{C}_{q}\right) \cdot W_{q}
$$

On the other hand, for each finite word $u \in \Sigma^{\star}$, the set $u \cdot W_{q}$ is a meager $\Sigma_{2}^{0}$-set, since $W_{q}$ is a meager $\Sigma_{2}^{0}$-set. Thus the set

$$
F=\bigcup_{q \in K} L\left(\mathcal{C}_{q}\right) \cdot W_{q}
$$

is also a countable union of closed sets with empty interiors, since $K$ is finite and each language $L\left(\mathcal{C}_{q}\right)$ is countable. Moreover the $\omega$-language $F$ is regular.

We can now set $G_{n}=\Sigma^{\omega} \backslash F_{n}$ and $G=\bigcap_{n>1} G_{n}=\Sigma^{\omega} \backslash \bigcup_{n>1} F_{n}=\Sigma^{\omega} \backslash F$. Then $G$ is a countable intersection of dense open subsets of $\Sigma^{\omega}$, hence also a dense $\Pi_{2}^{0}$-subset $G$ of $\Sigma^{\omega}$. Moreover we can construct a deterministic Muller automaton and even a deterministic Büchi automaton (since $G$ is a $\boldsymbol{\Pi}_{2}^{0}$-set, see [18, page 41]) accepting $G$. We can now see that the restriction $f_{G}$ of the function $f$ to $G$ is continuous. This follows from the fact that the inverse image of every basic open set of $\Gamma^{\omega}$ by the function $f_{G}$ is an open subset of $G$ because for each integer $n \geq 1$, it holds that $f_{G}^{-1}\left(U_{n}\right)=f^{-1}\left(U_{n}\right) \cap G=V_{n} \cap G$.

Remark 24. The above dense $\Pi_{2}^{0}$-subset $G$ of $\Sigma^{\omega}$ is comeager and thus Theorem 23 shows that one can construct a deterministic Büchi automaton accepting a "big" $\omega$-rational subset of $\Sigma^{\omega}$ on which the function $f$ is continuous.

\section{Deciding the first Baire class and more ...}

Notice that in this section we consider total functions. We first give a definition of Baire class $n$ functions.

Definition 25 (see [15]). Let $X$ and $Y$ be metrizable spaces and $F: X \rightarrow Y$ be a function. Then $F$ is said to be of Baire class 0 if $F$ is continuous. The function $F$ is said to be of Baire class 1 if for every open subset $V$ of $Y$ the inverse image of $V$ by $F$ is a $\boldsymbol{\Sigma}_{2}^{0}$-subset of $X$. Then for every integer $n>1$ the function $F$ is said to be of Baire class $n$ if it is the pointwise limit of a sequence $\left(F_{k}\right)_{k \geq 0}$ of functions of Baire class $n-1$. 
If we only consider functions $F: \Sigma^{\omega} \rightarrow \Gamma^{\omega}$ from a Cantor space to another Cantor space we have another characterization of Baire class $n$ functions which is given by the following:

Theorem 26 (see Theorem 24.3 in [15]). Let $F: \Sigma^{\omega} \rightarrow \Gamma^{\omega}$ be a function from a Cantor space to another Cantor space. Then, for every integer $n \geq 0$, the function $F$ is of Baire class $n$ if and only if for every open subset $V \subseteq \Gamma^{\omega}$ the inverse image of $V$ by $F$ is in the Borel class $\boldsymbol{\Sigma}_{n+1}^{0}$.

On the other hand, a Cantor space $\Gamma^{\omega}$ has a countable basis of clopen (i.e. closed and open) sets formed by the collection $\left(u \cdot \Gamma^{\omega}\right)$ for $u \in \Gamma^{\star}$. Therefore we easily get the following equivalent characterization.

Corollary 27. Let $F: \Sigma^{\omega} \rightarrow \Gamma^{\omega}$ be a function from a Cantor space to another Cantor space. Then, for every integer $n \geq 0$, the function $F$ is of Baire class $n$ if and only if for every $u \in \Gamma^{\star}$ the inverse image of the clopen set $\left(u \cdot \Gamma^{\omega}\right)$ by $F$ is in the Borel class $\boldsymbol{\Delta}_{n+1}^{0}$.

Notice that we also have the following interesting link between Baire class 1 functions and the notion of continuity set of a function.

Theorem 28 (Baire, see [15]). Let $F: \Sigma^{\omega} \rightarrow \Gamma^{\omega}$ be a function. If the function $F$ is of Baire class 1 then the continuity set $C(F)$ of $F$ is a dense $\mathbf{\Pi}_{2}^{0}$-set.

If we now consider an $\omega$-rational function $F: \Sigma^{\omega} \rightarrow \Gamma^{\omega}$, then every basic open set $\left(u \cdot \Gamma^{\omega}\right)$ of $\Gamma^{\omega}$ is $\omega$-regular and this implies that $F^{-1}\left(u \cdot \Gamma^{\omega}\right)$ is also $\omega$-regular and hence $\boldsymbol{\Delta}_{3}^{0}$. Then we have the following proposition.

Proposition 29 ([3, 4]). Every $\omega$-rational function is of Baire class 2.

It is then very natural to ask whether one can decide whether a given $\omega$ rational function is of Baire class 1. Cagnard and Simonnet proved in [3] that one can decide whether a given synchronous $\omega$-rational function is of Baire class 1. Their proof used the notion of undergraph and overgraph of a function $F$ : $\Sigma^{\omega} \rightarrow \Gamma^{\omega}$, when the set $\Gamma^{\omega}$ is equipped with the lexicographic ordering.

Theorem 30 (Cagnard-Simonnet [3]). One can decide whether a given synchronous $\omega$-rational function, whose graph is accepted by a given Büchi or Muller automaton $\mathcal{A}$, is of Baire class 1.

We first give here a more direct automata theoretic proof of this result, which do not use the notions of undergraph and overgraph of a function.

Proof. Let $F: \Sigma^{\omega} \rightarrow \Gamma^{\omega}$ be a synchronous $\omega$-rational function whose graph $G$ is accepted by a given deterministic Muller automaton $\mathcal{A}=\left(K, \Sigma \times \Gamma, \delta, q_{0}, \mathcal{F}\right)$, where $\mathcal{F} \subseteq 2^{K}$ is here the collection of designated state sets.

As in the preceding section, for $q \in K$ we also denote $\mathcal{A}_{q}$ the automaton $\mathcal{A}$ in which we have changed the initial state so that the initial state of $\mathcal{A}_{q}$ is $q$ instead of $q_{0}$. 
Let then $u \in \Gamma^{\star}$. We are going to describe the set $F^{-1}\left(u \cdot \Gamma^{\omega}\right)$. We denote

$$
A_{u}=\left\{v \in \Sigma^{\star}|| v|=| u \mid \text { and }(v, u) \cdot\left(\Sigma^{\omega} \times \Gamma^{\omega}\right) \cap G \neq \emptyset\right\}
$$

Then for every $v \in A_{u}$, we denote $q_{(v, u)}$ the state in which the automaton $\mathcal{A}$ is after the reading of the pair $(v, u)$. The rational relation $R\left(\mathcal{A}_{q_{(v, u)}}\right) \subseteq \Sigma^{\omega} \times \Gamma^{\omega}$ is functional since the relation $R(\mathcal{A})$ is functional. Then $\operatorname{Dom}\left(R\left(\mathcal{A}_{q_{(v, u)}}\right)\right) \subseteq \Sigma^{\omega}$ is a regular $\omega$-language and one can construct a deterministic Muller automaton accepting it. It is now easy to see that

$$
F^{-1}\left(u \cdot \Gamma^{\omega}\right)=\bigcup_{v \in A_{u}} v \cdot \operatorname{Dom}\left(R\left(\mathcal{A}_{q_{(v, u)}}\right)\right)
$$

We can now notice that $F^{-1}\left(u \cdot \Gamma^{\omega}\right)$ is a $\boldsymbol{\Delta}_{2}^{0}$-set iff for all $v \in A_{u}$ it holds that $\operatorname{Dom}\left(R\left(\mathcal{A}_{q_{(v, u)}}\right)\right)$ is a $\boldsymbol{\Delta}_{2}^{0}$-set:

Firstly, if for every $v \in A_{u}$ it holds that $\operatorname{Dom}\left(R\left(\mathcal{A}_{q_{(v, u)}}\right)\right)$ is a $\boldsymbol{\Delta}_{2}^{0}$-set then $v \cdot \operatorname{Dom}\left(R\left(\mathcal{A}_{q_{(v, u)}}\right)\right)$ is also a $\boldsymbol{\Delta}_{2}^{0}$-set and therefore by finite union $F^{-1}\left(u \cdot \Gamma^{\omega}\right)$ is a $\boldsymbol{\Delta}_{2}^{0}$-set. (Notice that this includes the particular case where $A_{u}=\emptyset$ and then $F^{-1}\left(u \cdot \Gamma^{\omega}\right)=\emptyset$ is a $\boldsymbol{\Delta}_{2}^{0}$-set. $)$

Secondly, if $F^{-1}\left(u \cdot \Gamma^{\omega}\right)$ is a $\Delta_{2}^{0}$-set then for every $v \in A_{u}$ the set $F^{-1}\left(u \cdot \Gamma^{\omega}\right) \cap$ $v \cdot \Gamma^{\omega}=v \cdot \operatorname{Dom}\left(R\left(\mathcal{A}_{q_{(v, u)}}\right)\right)$ is the intersection of a $\boldsymbol{\Delta}_{2}^{0}$-set and of an open set hence also a $\boldsymbol{\Delta}_{2}^{0}$-set. Moreover " $v \cdot \operatorname{Dom}\left(R\left(\mathcal{A}_{q_{(v, u)}}\right)\right)$ is a $\boldsymbol{\Delta}_{2}^{0}$-set" easily implies that $\operatorname{Dom}\left(R\left(\mathcal{A}_{q_{(v, u)}}\right)\right)$ is a $\boldsymbol{\Delta}_{2}^{0}$-set.

To conclude it suffices now to remark that the function $F$ is of Baire class 1 iff for all states $q$ of $\mathcal{A}$ which are accessible from $q_{0}$ (when reading some pair $(v, u))$ it holds that $\operatorname{Dom}\left(R\left(\mathcal{A}_{q}\right)\right)$ is a $\boldsymbol{\Delta}_{2}^{0}$-set. This is clearly decidable since one can decide whether a regular $\omega$-language is a $\boldsymbol{\Delta}_{2}^{0}$-set.

From the above proof we are now going to show that one can also easily obtain an extension of Theorem 30 based on the notion of the Wadge hierarchy which is a great refinement of the Borel hierarchy, firstly defined by Wadge via reductions by continuous functions [30].

We first now recall the notion of Wadge reduction and Wadge hierarchy on a Cantor space.

Definition 31 (Wadge [30]). Let $X, Y$ be two finite alphabets. For $L \subseteq X^{\omega}$ and $L^{\prime} \subseteq Y^{\omega}, L$ is said to be Wadge reducible to $L^{\prime}\left(L \leq_{W} L^{\prime}\right)$ iff there exists a continuous function $f: X^{\omega} \rightarrow Y^{\omega}$, such that $L=f^{-1}\left(L^{\prime}\right)$. The sets $L$ and $L^{\prime}$ are Wadge equivalent iff $L \leq_{W} L^{\prime}$ and $L^{\prime} \leq_{W} L$. This will be denoted by $L \equiv_{W} L^{\prime}$. And we shall say that $L<_{W} L^{\prime}$ iff $L \leq_{W} L^{\prime}$ but not $L^{\prime} \leq_{W} L$.

$A$ set $L \subseteq X^{\omega}$ is said to be self dual iff $L \equiv_{W} L^{-}$, and otherwise it is said to be non self dual.

The relation $\leq_{W}$ is reflexive and transitive, and $\equiv_{W}$ is an equivalence relation. The equivalence classes of $\equiv_{W}$ are called Wadge degrees. The Wadge hierarchy $W H$ is the class of Borel subsets of a set $X^{\omega}$, where $X$ is a finite set, equipped with $\leq_{W}$ and with $\equiv_{W}$. 
For $L \subseteq X^{\omega}$ and $L^{\prime} \subseteq Y^{\omega}$, if $L \leq_{W} L^{\prime}$ and $L=f^{-1}\left(L^{\prime}\right)$ where $f$ is a continuous function from $X^{\omega}$ into $Y^{\omega}$, then $f$ is called a continuous reduction of $L$ to $L^{\prime}$. Intuitively it means that $L$ is less complicated than $L^{\prime}$ because to check whether $x \in L$ it suffices to check whether $f(x) \in L^{\prime}$ where $f$ is a continuous function. Hence the Wadge degree of an $\omega$-language is a measure of its topological complexity. The Wadge class of a set $L \subseteq X^{\omega}$ is equal to $[L]=\left\{L^{\prime} \subseteq Y^{\omega}\right.$ | $Y$ is a finite alphabet and $\left.L^{\prime} \leq_{W} L\right\}$.

Recall that each Borel class $\Sigma_{n}^{0}$ and $\boldsymbol{\Pi}_{n}^{0}$ is a Wadge class.

Notice that in the above definition, we consider that a subset $L \subseteq X^{\omega}$ is given together with the alphabet $X$.

There is a close relationship between Wadge reducibility and the notion of a particular class of infinite 2-player games now called Wadge games.

Definition 32. Let $L \subseteq X^{\omega}$ and $L^{\prime} \subseteq Y^{\omega}$. The Wadge game $W\left(L, L^{\prime}\right)$ is a game with perfect information between two players, player 1 who is in charge of $L$ and player 2 who is in charge of $L^{\prime}$. Player 1 first writes a letter $a_{1} \in X$, then player 2 writes a letter $b_{1} \in Y$, then player 1 writes a letter $a_{2} \in X$, and so on. The two players alternatively write letters $a_{n}$ of $X$ for player 1 and $b_{n}$ of $Y$ for player 2. After $\omega$ steps, the player 1 has written an $\omega$-word $a \in X^{\omega}$ and the player 2 has written an $\omega$-word $b \in Y^{\omega}$. The player 2 is allowed to skip, even infinitely often, provided he really writes an $\omega$-word in $\omega$ steps. The player 2 wins the play iff $\left[a \in L \leftrightarrow b \in L^{\prime}\right]$, i.e. iff :

$$
\text { [( } \left.\left.a \in L \text { and } b \in L^{\prime}\right) \text { or } \quad\left(a \notin L \text { and } b \notin L^{\prime} \text { and } b \text { is infinite }\right)\right] \text {. }
$$

Recall that a strategy for player 1 is a function $\sigma:(Y \cup\{s\})^{\star} \rightarrow X$. And a strategy for player 2 is a function $f: X^{+} \rightarrow Y \cup\{s\}$. The strategy $\sigma$ is a winning stategy for player 1 iff he always wins a play when he uses the strategy $\sigma$, i.e. when the $n^{t h}$ letter he writes is given by $a_{n}=\sigma\left(b_{1} \cdots b_{n-1}\right)$, where $b_{i}$ is the letter written by player 2 at step $i$ and $b_{i}=s$ if player 2 skips at step $i$.A winning strategy for player 2 is defined in a similar manner.

Martin's Theorem states that every Gale-Stewart game $G(X)$ (see [15]), with $X$ a Borel set, is determined and this implies the following :

Theorem 33 (Wadge). Let $L \subseteq X^{\omega}$ and $L^{\prime} \subseteq Y^{\omega}$ be two Borel sets, where $X$ and $Y$ are finite alphabets. Then the Wadge game $W\left(L, L^{\prime}\right)$ is determined: one of the two players has a winning strategy. And $L \leq_{W} L^{\prime}$ iff the player 2 has a winning strategy in the game $W\left(L, L^{\prime}\right)$.

Theorem 34 (Wadge). Up to the complement and $\equiv_{W}$, the class of Borel subsets of $X^{\omega}$, for a finite alphabet $X$ having at least two letters, is a well ordered hierarchy. There is an ordinal $|W H|$, called the length of the hierarchy, and a map $d_{W}^{0}$ from $W H$ onto $|W H|-\{0\}$, such that for all $L, L^{\prime} \subseteq X^{\omega}$ :

$d_{W}^{0} L<d_{W}^{0} L^{\prime} \leftrightarrow L<_{W} L^{\prime}$ and

$d_{W}^{0} L=d_{W}^{0} L^{\prime} \leftrightarrow\left[L \equiv_{W} L^{\prime}\right.$ or $\left.L \equiv_{W} L^{\prime-}\right]$.

The Wadge hierarchy of Borel sets of finite rank has length ${ }^{1} \varepsilon_{0}$ where ${ }^{1} \varepsilon_{0}$ is the limit of the ordinals $\alpha_{n}$ defined by $\alpha_{1}=\omega_{1}$ and $\alpha_{n+1}=\omega_{1}^{\alpha_{n}}$ for $n$ a non 
negative integer, $\omega_{1}$ being the first non countable ordinal. Then ${ }^{1} \varepsilon_{0}$ is the first fixed point of the ordinal exponentiation of base $\omega_{1}$.

The Wadge hierarchy of $\boldsymbol{\Delta}_{2}^{0}$-sets has length $\omega_{1}$ and the length of the Wadge hierarchy of boolean combinations of $\boldsymbol{\Pi}_{2}^{0}$-sets is equal to the ordinal $\omega_{1}^{\omega}$.

On the other hand, the trace of the Wadge hierarchy on the $\omega$-regular languages is called the Wagner hierarchy. It has been completely described by Wagner in [31]. Its length is the (countable) ordinal $\omega^{\omega}$. Wagner gave an automatonlike characterization of this hierarchy, based on the notions of chain and superchain, together with an algorithm to compute the Wadge (Wagner) degree of any given $\omega$-regular language, see also $[21,23,22,24]$.

We now see that we can get a refinement of the Baire hierarchy of functions, using the notion of the Wadge hierarchy. Recall that a function $F: \Sigma^{\omega} \rightarrow \Gamma^{\omega}$ is of Baire class $n$ if and only if for every $u \in \Gamma^{\star}$ the inverse image of the clopen set $\left(u \cdot \Gamma^{\omega}\right)$ by $F$ is in the Borel class $\boldsymbol{\Delta}_{n+1}^{0}$. One can refine this notion by considering, for each Wadge class $[L] \subseteq \boldsymbol{\Delta}_{n+1}^{0}$, the class $\mathcal{C}_{[L]}$ of functions $F: \Sigma^{\omega} \rightarrow \Gamma^{\omega}$ such that for every $u \in \Gamma^{\star}$ the inverse image of the clopen set $\left(u \cdot \Gamma^{\omega}\right)$ by $F$ is in the Wadge class $[L]$.

We can now state the following refinement of Theorem 30.

Theorem 35. For a regular $\omega$-language L accepted by a given Büchi or Muller automaton and a synchronous $\omega$-rational function $F$, whose graph is accepted by a given Büchi or Muller automaton, one can decide whether $F$ belongs to the class $\mathcal{C}_{[L]}$.

Proof. Let, as in the above proof of Theorem 30, $F: \Sigma^{\omega} \rightarrow \Gamma^{\omega}$ be a synchronous $\omega$-rational function whose graph $G$ is accepted by a deterministic Muller automaton over the product alphabet $\Sigma \times \Gamma$. Recall that, for $u \in \Gamma^{\star}$, we got the following description of the set $F^{-1}\left(u \cdot \Gamma^{\omega}\right)$.

$$
F^{-1}\left(u \cdot \Gamma^{\omega}\right)=\bigcup_{v \in A_{u}} v \cdot \operatorname{Dom}\left(R\left(\mathcal{A}_{q_{(v, u)}}\right)\right)
$$

We can now determine the Wadge degree of $F^{-1}\left(u \cdot \Gamma^{\omega}\right)$. We first notice that we can determine the Wadge-Wagner degrees of every $\omega$-language $\operatorname{Dom}\left(R\left(\mathcal{A}_{q_{(v, u)}}\right)\right)$ since for every state $q \in K$ we can construct a deterministic Muller automaton $\mathcal{B}_{q}$ accepting the $\omega$-language $\operatorname{Dom}\left(R\left(\mathcal{A}_{q}\right)\right)$. We now consider the following cases.

Case 1. $u=\varepsilon$. In that case it holds that $F^{-1}\left(u \cdot \Gamma^{\omega}\right)=F^{-1}\left(\Gamma^{\omega}\right)=\Sigma^{\omega}$.

Case 2. $u \neq \varepsilon$ and $A_{u}=\emptyset$. In that case $F^{-1}\left(u \cdot \Gamma^{\omega}\right)=\emptyset$.

Case 3. $u \neq \varepsilon$ and $F^{-1}\left(u \cdot \Gamma^{\omega}\right)=\Sigma^{\omega}$.

Case 4. $u \neq \varepsilon$ and there is some $v_{0} \in A_{u}$ such that for every $v \in A_{u}$ it holds that $\operatorname{Dom}\left(R\left(\mathcal{A}_{q_{(v, u)}}\right)\right) \leq_{W} \operatorname{Dom}\left(R\left(\mathcal{A}_{\left.q_{\left(v_{0}, u\right.}\right)}\right)\right) \neq \Sigma^{\omega}$. (Notice that this implies in particular that $F^{-1}\left(u \cdot \Gamma^{\omega}\right) \neq \Sigma^{\omega}$.)

In that case we prove that $F^{-1}\left(u \cdot \Gamma^{\omega}\right) \equiv_{W} \cdot \operatorname{Dom}\left(R\left(\mathcal{A}_{q_{\left(v_{0}, u\right)}}\right)\right)$. 
Consider firstly the Wadge game $W\left(F^{-1}\left(u \cdot \Gamma^{\omega}\right), \operatorname{Dom}\left(R\left(\mathcal{A}_{q_{\left(v_{0}, u\right)}}\right)\right)\right.$. We claim that Player 2 has a winning strategy in this game. Player 2, following this strategy, begins by skipping his turn until Player 1 has written a word $v$ of length $|u|$. Then if $v \notin A_{u}$ then Player 1 is now like a player in charge of the emptyset therefore at the end of the play the infinite word written by Player 1 will be surely outside her (empty) set. Thus Player 2 can write in $\omega$ steps an infinite word which is in $\Sigma^{\omega} \backslash \operatorname{Dom}\left(R\left(\mathcal{A}_{q_{\left(v_{0}, u\right)}}\right)\right.$ so that he wins the play. And if $v \in A_{u}$ then Player 1 is now like a player in charge of the set $\operatorname{Dom}\left(R\left(\mathcal{A}_{q_{(v, u)}}\right)\right)$ and Player 2 can win the play since by hypothesis $\operatorname{Dom}\left(R\left(\mathcal{A}_{q_{(v, u)}}\right)\right) \leq_{W} \operatorname{Dom}\left(R\left(\mathcal{A}_{q_{\left(v_{0}, u\right)}}\right)\right)$. This shows that $F^{-1}\left(u \cdot \Gamma^{\omega}\right) \leq_{W} \operatorname{Dom}\left(R\left(\mathcal{A}_{q_{\left(v_{0}, u\right)}}\right)\right.$.

Secondly consider the Wadge game $W\left(\operatorname{Dom}\left(R\left(\mathcal{A}_{q_{\left(v_{0}, u\right)}}\right), F^{-1}\left(u \cdot \Gamma^{\omega}\right)\right)\right.$. We claim that Player 2 has a winning strategy in this game. Indeed Player 2 can first write $v_{0}$ in the first $|u|$ steps of the play. Then Player 2 just follows what has written Player 1 so that at the end of the play Player 1 has written an $\omega$-word $x$ and Player 2 has written an $\omega$-word $v_{0} \cdot x$ and Player 2 wins the play.

Finally $F^{-1}\left(u \cdot \Gamma^{\omega}\right) \equiv_{W} \operatorname{Dom}\left(R\left(\mathcal{A}_{q_{\left(v_{0}, u\right)}}\right)\right)$.

Case 5. $u \neq \varepsilon$ and $F^{-1}\left(u \cdot \Gamma^{\omega}\right) \neq \Sigma^{\omega}$ and there is some $v_{0} \in A_{u}$ such that for every $v \in A_{u}$ it holds that $\operatorname{Dom}\left(R\left(\mathcal{A}_{q_{(v, u)}}\right)\right) \leq_{W} \operatorname{Dom}\left(R\left(\mathcal{A}_{q_{\left(v_{0}, u\right)}}\right)\right)=\Sigma^{\omega}$.

Then for every $v \in A_{u}$ it holds that $\operatorname{Dom}\left(R\left(\mathcal{A}_{q_{(v, u)}}\right)\right)=\Sigma^{\omega}$ since the set $\Sigma^{\omega}$ is at the bottom of the Wadge hierarchy and there are no other sets below it. Thus $F^{-1}\left(u \cdot \Gamma^{\omega}\right)=\bigcup_{v \in A_{u}} v \cdot \Sigma^{\omega}$ and $A_{u} \subsetneq\left\{v \in \Sigma^{\star}|| v|=| u \mid\right\}$ since $F^{-1}\left(u \cdot \Gamma^{\omega}\right) \neq \Sigma^{\omega}$. In that case $F^{-1}\left(u \cdot \Gamma^{\omega}\right)$ is a clopen set (different from the emptyset and from the whole set $\Sigma^{\omega}$ and just above them in the Wadge hierarchy on the space $\Sigma^{\omega}$ ) of Wadge degree 2 .

Case 6. $u \neq \varepsilon$ and there exist $v_{0}, v_{0}^{\prime} \in A_{u}$ such that for every $v \in A_{u}$ it holds that $\operatorname{Dom}\left(R\left(\mathcal{A}_{q_{(v, u)}}\right)\right) \leq_{W} \operatorname{Dom}\left(R\left(\mathcal{A}_{q_{\left(v_{0}, u\right)}}\right)\right)$ or $\operatorname{Dom}\left(R\left(\mathcal{A}_{q_{(v, u)}}\right)\right) \leq_{W}$ $\operatorname{Dom}\left(R\left(\mathcal{A}_{q_{\left(v_{0}^{\prime}, u\right)}}\right)\right)$. We also asssume that $\operatorname{Dom}\left(R\left(\mathcal{A}_{q_{\left(v_{0}, u\right)}}\right)\right)$ is non self-dual and $\operatorname{Dom}\left(R\left(\mathcal{A}_{q_{\left(v_{0}, u\right)}}\right)\right) \equiv_{W} \operatorname{Dom}\left(R\left(\mathcal{A}_{q_{\left(v_{0}^{\prime}, u\right)}}\right)\right)^{-}$. (Notice that this implies in particular that $F^{-1}\left(u \cdot \Gamma^{\omega}\right) \neq \Sigma^{\omega}$ since $\operatorname{Dom}\left(R\left(\mathcal{A}_{q_{\left(v_{0}, u\right)}}\right)\right) \neq \Sigma^{\omega}$ and $\operatorname{Dom}\left(R\left(\mathcal{A}_{q_{\left(v_{0}^{\prime}, u\right)}}\right)\right) \neq$ $\left.\Sigma^{\omega}.\right)$

In that case $F^{-1}\left(u \cdot \Gamma^{\omega}\right) \equiv_{W} a \cdot \operatorname{Dom}\left(R\left(\mathcal{A}_{q_{\left(v_{0}, u\right)}}\right)\right) \cup b \cdot \operatorname{Dom}\left(R\left(\mathcal{A}_{q_{\left(v_{0}^{\prime}, u\right)}}\right)\right)$, where $a$ and $b$ are two distinct letters of $\Sigma$. Then $F^{-1}\left(u \cdot \Gamma^{\omega}\right)$ belongs to the first self-dual degree just above the non self-dual degrees of $\operatorname{Dom}\left(R\left(\mathcal{A}_{q_{\left(v_{0}, u\right)}}\right)\right)$ and $\operatorname{Dom}\left(R\left(\mathcal{A}_{q_{\left(v_{0}^{\prime}, u\right)}}\right)\right)$. This can be proved using Wadge games in a very similar way as in the above case 4 . Details are here left to the reader.

Notice that these cases $1-6$ are exhaustive since the Wadge-Wagner hierarchy is, up to complement and $\equiv_{W}$, a well ordered hierarchy.

We are now going to determine the smaller Wadge class [T] such that $F$ belongs to the class $\mathcal{C}_{[T]}$.

Case 1 shows that there is some $u(u=\varepsilon)$ such that $F^{-1}\left(u \cdot \Gamma^{\omega}\right)=\Sigma^{\omega}$. Moreover it is not possible that Case 2 occurs for all $u \neq \varepsilon$ since there is at least one $\omega$-word in the range of $F$. It is also not possible that Case 3 occurs for all 
$u \neq \varepsilon$ because if for some $u \neq \varepsilon$ it holds that $F^{-1}\left(u \cdot \Gamma^{\omega}\right)=\Sigma^{\omega}$ then for $v \in \Gamma^{\star}$, $|v|=|u|$, it will hold that $F^{-1}\left(v \cdot \Gamma^{\omega}\right)=\emptyset$.

If only Cases $1-3$ occur it is easy to see that the function $F$ is actually constant (and in that case its range contains only an ultimately periodic word since the range is a regular $\omega$-language). Indeed if the image of $F$ contains (at least) two $\omega$-words $x$ and $y$ then there is some integer $n \geq 1$ such that $x[n] \neq y[n]$. Then $F^{-1}\left(x[n] \cdot \Gamma^{\omega}\right)$ and $F^{-1}\left(y[n] \cdot \Gamma^{\omega}\right)$ are both different from the empty set and from the whole set $\Sigma^{\omega}$. Notice that we can construct a Büchi or Muller automaton accepting the range of $F$ and decide whether it is a singleton. If this is the case then for $u \in \Gamma^{\star}$, the set $F^{-1}\left(u \cdot \Gamma^{\omega}\right)$ can be either empty or equal to $\Sigma^{\omega}$. And the least Wadge class above the two non self dual degrees of $\emptyset$ and of $\Sigma^{\omega}$ is the class of clopen sets which can be written $\left[a \cdot \Sigma^{\omega}\right]$ for some letter $a \in \Sigma$.

If on the contrary the function $F$ is not constant then cases 4 or 5 or 6 may appear. Then we begin by determining the Wadge-Wagner degrees of the regular $\omega$-languages $\operatorname{Dom}\left(R\left(\mathcal{A}_{q}\right)\right)$, for all states $q$ of $\mathcal{A}$ which are accessible from $q_{0}$ (when reading some pair $(v, u)$ ) and such that $R\left(\mathcal{A}_{q}\right) \neq \emptyset$.

If all these sets $\operatorname{Dom}\left(R\left(\mathcal{A}_{q}\right)\right)$ are equal to $\Sigma^{\omega}$ then only case 5 may appear. In that case we have seen that $F^{-1}\left(u \cdot \Gamma^{\omega}\right)$ is a clopen set (different from the empty set and from the whole set $\Sigma^{\omega}$ ). And since cases 4 and 6 cannot appear, then the function $F$ belongs to the class $\mathcal{C}_{\left[a \cdot \Sigma^{\omega}\right]}$ for any letter $a \in \Sigma$.

Assume now that there is a state $q_{1} \in K$ (accessible from $\left.q_{0}\right)$ with $R\left(\mathcal{A}_{q_{1}}\right) \neq \emptyset$ and such that for all other states $q \in K$ which are accessible from $q_{0}$ with $R\left(\mathcal{A}_{q}\right) \neq \emptyset$ it holds that $\operatorname{Dom}\left(R\left(\mathcal{A}_{q}\right)\right) \leq_{W} \operatorname{Dom}\left(R\left(\mathcal{A}_{q_{1}}\right)\right) \neq \Sigma^{\omega}$. Then we can deduce from the above cases $4-6$ that there is some $u \in \Gamma^{\star}$ such that $F^{-1}\left(u \cdot \Gamma^{\omega}\right) \equiv_{W} \operatorname{Dom}\left(R\left(\mathcal{A}_{q_{1}}\right)\right.$. Moreover this implies that $\left[\operatorname{Dom}\left(R\left(\mathcal{A}_{q_{1}}\right)\right]\right.$ is the least Wadge class $[\mathrm{T}]$ such that $F$ belongs to the class $\mathcal{C}_{[T]}$.

Assume now that there are some states $q_{1} \in K$ and $q_{2} \in K$ (accessible from $\left.q_{0}\right)$ with $R\left(\mathcal{A}_{q_{1}}\right) \neq \emptyset, R\left(\mathcal{A}_{q_{2}}\right) \neq \emptyset$ and such that for all other states $q \in K$ which are accessible from $q_{0}$ with $R\left(\mathcal{A}_{q}\right) \neq \emptyset$ it holds that $\operatorname{Dom}\left(R\left(\mathcal{A}_{q}\right)\right) \leq_{W}$ $\operatorname{Dom}\left(R\left(\mathcal{A}_{q_{1}}\right)\right) \neq \Sigma^{\omega}$ or $\operatorname{Dom}\left(R\left(\mathcal{A}_{q}\right)\right) \leq_{W} \operatorname{Dom}\left(R\left(\mathcal{A}_{q_{2}}\right)\right) \neq \Sigma^{\omega}$. Moreover we assume that $\operatorname{Dom}\left(R\left(\mathcal{A}_{q_{1}}\right)\right)$ and $\operatorname{Dom}\left(R\left(\mathcal{A}_{q_{2}}\right)\right)$ are non self-dual and that $\operatorname{Dom}\left(R\left(\mathcal{A}_{q_{1}}\right)\right) \equiv \operatorname{Dom}\left(R\left(\mathcal{A}_{q_{2}}\right)\right)^{-}$. Then we can deduce from the above cases 4 and 6 that either there are some $u_{1}, u_{2} \in \Gamma^{\star}$ such that $F^{-1}\left(u_{1} \cdot \Gamma^{\omega}\right) \equiv_{W}$ $\operatorname{Dom}\left(R\left(\mathcal{A}_{q_{1}}\right)\right.$ and $F^{-1}\left(u_{2} \cdot \Gamma^{\omega}\right) \equiv_{W} \operatorname{Dom}\left(R\left(\mathcal{A}_{q_{2}}\right)\right.$ or there is some $u \in \Sigma^{\omega}$ such that $F^{-1}\left(u \cdot \Gamma^{\omega}\right) \equiv a \cdot \operatorname{Dom}\left(R\left(\mathcal{A}_{q_{1}}\right)\right) \cup b \cdot \operatorname{Dom}\left(R\left(\mathcal{A}_{q_{2}}\right)\right)$, where $a$ and $b$ are two distinct letters of $\Sigma$. In both cases we see that $\left[a \cdot \operatorname{Dom}\left(R\left(\mathcal{A}_{q_{1}}\right)\right) \cup b \cdot \operatorname{Dom}\left(R\left(\mathcal{A}_{q_{2}}\right)\right)\right]$ is the least Wadge class $[T]$ such that $F$ belongs to the class $\mathcal{C}_{[T]}$.

Finally we have determined the least Wadge class $[T]$ such that $F$ belongs to the class $\mathcal{C}_{[T]}$. Moreover we have found a regular $\omega$-language $R$ such that $[T]=[R]$. On the other hand, we know that the Wadge-Wagner hierarchy is effective therefore we can decide, for a given regular $\omega$-language $L$, whether the function $F$ belongs to the class $\mathcal{C}_{[L]}$. 


\section{Concluding remarks}

We have proved some new effective properties, related to the points of continuity, of $\omega$-rational functions. We hope these properties will be useful for further studies involving $\omega$-rational functions. For instance an $\omega$-automatic structure is defined via synchronous infinitary rational relations, see $[2,16]$. On the other hand, any (synchronous) infinitary rational relation is uniformizable by a (synchronous) $\omega$-rational function, see [6]. Thus we can expect that our results will be useful in particular in the study of $\omega$-automatic structures.

We have got a new simple proof of the decidability of the Baire class 1 synchronous $\omega$-rational functions, and showed that one can decide, for a given synchronous $\omega$-rational function $F: \Sigma^{\omega} \rightarrow \Gamma^{\omega}$, whether the inverse image by $F$ of any basic open subset of $\Gamma^{\omega}$ is in a given Wadge class of the Wadge-Wagner hierarchy of $\omega$-regular sets. This provides a refinement of Cagnard and Simonnet's result. The case of asynchronous $\omega$-rational functions is left for further study.

\section{References}

1. Béal, M.P., Carton, O., Prieur, C., Sakarovitch, J.: Squaring transducers: an efficient procedure for deciding functionality and sequentiality. Theoretical Computer Science 292(1), 45-63 (2003)

2. Blumensath, A., Grädel, E.: Finite presentations of infinite structures: Automata and interpretations. Theory of Computing Systems 37(6), 641-674 (2004)

3. Cagnard, B., Simonnet, P.: Automata, Borel functions and real numbers in Pisot base. Theoretical Informatics and Applications 41(1), 27-44 (2007)

4. Cagnard, B., Simonnet, P.: Baire and automata. Discrete Mathematics and Theoretical Computer Science. DMTCS. 9(2), 255-295 (2007)

5. Carton, O., Finkel, O., Simonnet, P.: On the continuity set of an omega rational function. Theoretical Informatics and Applications 42(1), 183-196 (2008)

6. Choffrut, C., Grigorieff, S.: Uniformization of rational relations. In: Karhumäki, J., Maurer, H.A., Paun, G., Rozenberg, G. (eds.) Jewels are Forever, Contributions on Theoretical Computer Science in Honor of Arto Salomaa. pp. 59-71. Springer (1999)

7. Diekert, V., Muscholl, A., Walukiewicz, I.: A note on monitors and Büchi automata. In: Leucker, M., Rueda, C., Valencia, F.D. (eds.) Theoretical Aspects of Computing - ICTAC 2015 - 12th International Colloquium Cali, Colombia, October 29-31, 2015, Proceedings. Lecture Notes in Computer Science, vol. 9399, pp. 39-57. Springer (2015)

8. Finkel, O.: Highly undecidable problems for infinite computations. RAIROTheoretical Informatics and Applications 43(2), 339-364 (2009)

9. Finkel, O.: Three applications to rational relations of the high undecidability of the infinite Post correspondence problem in a regular $\omega$-language. International Journal of Foundations of Computer Science 23(7), 1481-1498 (2012)

10. Finkel, O.: The automatic Baire property and an effective property of omegarational functions. In: Leporati, A., Martín-Vide, C., Shapira, D., Zandron, C. (eds.) Language and Automata Theory and Applications - 14th International Conference, LATA 2020, Milan, Italy, March 4-6, 2020, Proceedings. Lecture Notes in Computer Science, vol. 12038, pp. 303-314. Springer (2020) 
11. Frougny, C., Sakarovitch, J.: Synchronized rational relations of finite and infinite words. Theoretical Computer Science 108(1), 45-82 (1993)

12. Gire, F.: Relations rationnelles infinitaires. Ph.D. thesis, Université Paris VII (1981)

13. Gire, F.: Two decidability problems for infinite words. Information Processing Letters 22(3), 135-140 (1986)

14. Gire, F., Nivat, M.: Relations rationnelles infinitaires. Calcolo XXI, 91-125 (1984)

15. Kechris, A.S.: Classical descriptive set theory. Springer-Verlag, New York (1995)

16. Kuske, D., Lohrey, M.: First-order and counting theories of omega-automatic structures. Journal of Symbolic Logic 73(1), 129-150 (2008)

17. Michalewski, H., Mio, M., Skrzypczak, M.: Monadic second order logic with measure and category quantifiers. Logical Methods in Computer Science 14(2) (2018)

18. Perrin, D., Pin, J.E.: Infinite words, automata, semigroups, logic and games, Pure and Applied Mathematics, vol. 141. Elsevier (2004)

19. Prieur, C.: How to decide continuity of rational functions on infinite words. Theoretical Computer Science 250(1-2), 71-82 (2001)

20. Prieur, C.: How to decide continuity of rational functions on infinite words. Theoretical Computer Science 276(1-2), 445-447 (2002)

21. Selivanov, V.: Fine hierarchy of regular $\omega$-languages,. Theoretical Computer Science 191, 37-59 (1998)

22. Selivanov, V.: Fine hierarchies and m-reducibilities in theoretical computer science. Theoretical Computer Science 405(1-2), 116-163 (2008)

23. Selivanov, V.: Wadge reducibility and infinite computations. Special Issue on Intensional Programming and Semantics in honour of Bill Wadge on the occasion of his 60th cycle, Mathematics in Computer Science 2(1), 5-36 (2008)

24. Simonnet, P.: Automates et théorie descriptive. Ph.D. thesis, Université Paris VII (1992)

25. Staiger, L.: Reguläre nullmengen. J. Inf. Process. Cybern. 12(6), 307-311 (1976)

26. Staiger, L.: $\omega$-languages. In: Handbook of formal languages, Vol. 3, pp. 339-387. Springer, Berlin (1997)

27. Staiger, L.: Rich $\omega$-words and monadic second-order arithmetic. In: Computer science logic (Aarhus, 1997), pp. 478-490. Springer, Berlin (1998)

28. Thomas, W.: Automata on infinite objects. In: van Leeuwen, J. (ed.) Handbook of Theoretical Computer Science, vol. B, Formal models and semantics, pp. 135-191. Elsevier (1990)

29. Völzer, H., Varacca, D.: Defining fairness in reactive and concurrent systems. Journal of the ACM 59(3), 13:1-13:37 (2012)

30. Wadge, W.: Reducibility and determinateness in the Baire space. Ph.D. thesis, University of California, Berkeley (1983)

31. Wagner, K.: On $\omega$-regular sets. Information and Control 43(2), 123-177 (1979) 